\title{
AVALIAÇÃO DE ALIMENTADORES PARA ABELHA MANDAÇAIA (MELIPONA QUADRIFASCIATA ANTHIDIOIDES)
}

\author{
EVALUATION OF DEVICES FOR FEEDING MANDAÇAIA \\ (MELIPONA QUADRIFASCIATA ANTHIDIOIDES)
}

\author{
Sampaio, R.B. ${ }^{1 *}$; Paula-Leite, M.C. ${ }^{1 \mathrm{~A}} ;$ Carvalho, C.A.L. ${ }^{1 \mathrm{~B}}$ e Faquinello, P. ${ }^{1 \mathrm{C}}$ \\ ${ }^{1}$ Universidade Federal do Recôncavo da Bahia-UFRB. Centro de Ciências Agrárias, Ambientais e \\ Biológicas. Cruz das Almas-BA. Brasil. *robertosampaio@hotmail.com; Ameibydepaula@hotmail.com; \\ Bcalfredo@ufrb.edu.br; Cpatynello@gmail.com
}

\section{PalaVRas ChaVE ADICIONAIS \\ Abelha sem ferrão. Alimentação energética.}

\section{RESUMO}

Este trabalho teve por objetivo avaliar diferentes dispositivos para alimentação de colônias de abelha mandaçaia. Foram avaliados três alimentadores, Eiratama, Pernambucano e Prato, para a alimentação energética. Foram utilizados 5 dispositivos por tratamento, totalizando 15 colônias. Foram avaliados à capacidade de coleta e tempo até o consumo total do alimento fornecido. O Prato apresentou média para consumo de alimento de $13,55 \mathrm{~mL}$, sendo superior aos demais. Concluiu-se que, para a alimentação da abelha mandaçaia o Prato foi o mais eficiente.

\section{SUMMARY}

Three devices for feeding mandaçaia bee, for easy handling and efficiency use: Eiratama, Pernambucano and Prato, were evaluated. Five devices for treatment and 15 colonies total were used, testing the collect capacity and time spent to total consumption. The Prato device showed mean consumption of $13.55 \mathrm{~mL}$, being higher than the others. For feeding mandaçaia bee, the Prato type was the most efficient.

\section{INTRODUÇÃO}

O nordeste brasileiro possuiu grande instabilidade climática (Araújo e Souza 2011). A caatinga, vegetação predominante, é caracterizada por grande diversidade

\section{AdDITIONAL KEYWORDS \\ Energetic food. Stinglessbee.}

botânica propiciando floradas escalonadas durante o ano, independente da estação (Lima et al., 2012). Assim durante o período de escassez de recursos florais a alimentação artificial é uma das formas de manejo adotadas, uma vez que a vegetação nativa padece pelo desmatamento (Dias et al., 2010; Contrera etal., 2011).

Dentre as espécies de abelhas sem ferrão que ocorrem no Nordeste do Brasil, merece destaque o mel produzido pela espécie Melipona quadrifasciata anthidioides, conhecida por mandaçaia, por ser procurado pelo seu sabor, produção de até 2,0 litros por colônia em ambiente natural e de boa florada. Quando manejada adequadamente a produtividade por colônia pode aumentar, o que torna a criação desta espécie uma boa alternativa para agricultores.

Alves et al. (2007 e 2011) relatam que $M$. $q$. anthidioides aceita bem a alimentação artificial. Existem poucos trabalhos referentes à melhor forma de fornecimento do alimento energético para as abelhas sem ferrão, em especial a espécie mandaçaia. Este trabalho teve por objetivo avaliar diferentes alimentadores para colônias de mandaçaia quanto ao manuseio e uso. 

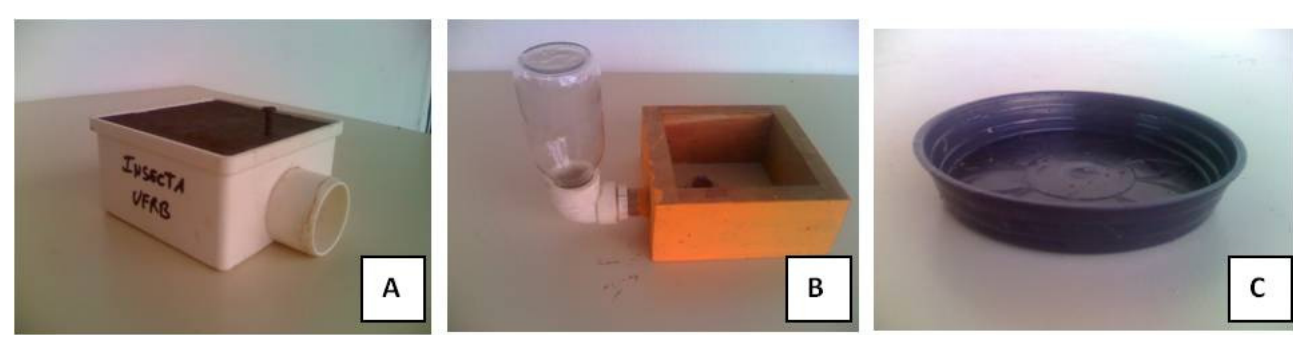

Figura 1. Alimentadores avaliados: Eiratama (A), Pernambucano $(B)$ e Prato $(C)$. (Devices evaluated: Eiratama (A), Pernambucano $(B)$ and Prato $(C)$ ).

\section{MATERIALE MÉTODOS}

O trabalho foi desenvolvido no meliponário do Grupo de Pesquisa Insecta do CCAAB/UFRB em Cruz das Almas - BA $\left(12^{\circ}\right.$ $39^{\prime} 20^{\prime \prime} \mathrm{We} 39^{\circ} 07^{\prime} 23^{\prime \prime} \mathrm{S}$, altitude $220 \mathrm{~m}$ ), em novembro de 2010 a maio de 2011.

As colônias estavam instaladas em caixa racional modelo INPA, consistindo de fundo $(17 \times 17 \mathrm{~cm})$, ninho (medida interna $13 \times 13 \times 6,5 \mathrm{~cm}$ ), sobreninho (medida interna $13 \times 13 \times 6,5 \mathrm{~cm})$, melgueira (medida interna $13 \times 13 \times 5 \mathrm{~cm})$ e tampa $(17 \times 17 \mathrm{~cm})$. As colônias foram selecionadas aleatoriamente, dentre as mais uniformes, de um universo de 20. Foi feito sorteio com relação aos tipos de alimentadores e adaptação de 30 dias.

Os alimentadores avaliados foram o Eiratama, Pernambucano e Prato (figura $1 \mathrm{e}$ 2), com 5 colônias avaliadas por tratamento.

No alimentador Eiratama foi utilizado uma caixa sifonada de banheiro, com a abertura interna voltada para cima e a própria tampa da caixa. Foram vedadas todas as frestas com fita adesiva. Sua instalação foi realizada por meio de um furo com cerra copo de $38 \mathrm{~mm}$ no lado contrário a entrada da colônia (figura 2).

No alimentador Pernambucano foi utilizado um joelho plástico de $32 \mathrm{~mm}$, com uma redução de $32 / 20,5 \mathrm{~cm}$ de tubo plástico de $20 \mathrm{~mm}$ e um joelho de $20 \mathrm{~mm}$. Para sua instalação foi realizado um furo com broca chata de $3 \backslash 4$ no lado contrário a entrada (figura 2). Foi tomado o cuidado para que este possua apenas $1 \mathrm{~cm}$ do fundo do ninho, para que a parte superior do joelho interno não ficasse muito alta e pudesse tocar no compartimento superior ou na tampa, impossibilitando a coleta do alimento pelas abelhas.

No alimentador Prato foi utilizado um
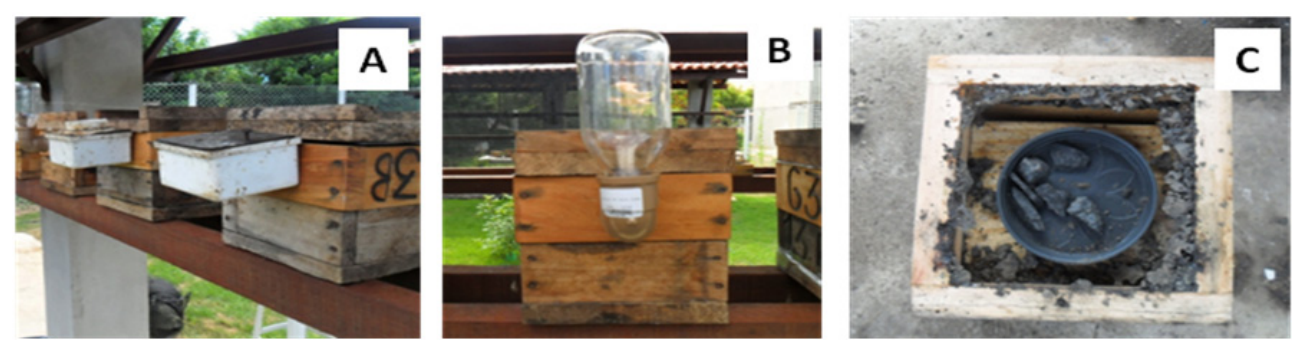

Figura 2. Alimentadores Eiratama (A), Pernambucano $(B)$ e Prato $(C)$ dispostos em colônias de mandaçaia alojadas em caixas INPA. (Devices Eiratama (A), Pernambucano (B) and Prato (C) arranged in colonies of mandaçaia estabilished in boxes INPA). 


\section{DISPOSITIVOS ALIMENTARESPARAABELHAMANDAÇAIA}

prato de caqueiro de $10 \mathrm{~cm}$, lixado, facilitando a aderência das abelhas, para que estas não se afogassem. Também foram colocadas algumas pedras tipo brita para facilitar a saída daquelas que caíssem no alimento (figura 2).

Em cada dispositivo foi utilizado como alimento um xarope, com $50 \%$ de água e $50 \%$ de açúcar, conforme metodologia adaptada de Alves et al. (2011), sendo aquecido para que o açúcar fosse totalmente dissolvido e fornecido em temperatura ambiente.

Os alimentadores foram avaliados quanto a coleta de alimento, em cada colônia. Foram fornecidos $30 \mathrm{~mL}$ do xarope/colônia, duas vezes por semana. $\mathrm{O}$ alimento foi fornecido às oito horas da manhã, mensurando o consumo a cada hora. Após quatro horas o consumo total de alimento foi quantificado.

As análises estatísticas foram realizadas utilizando o programa SAS (2007).

\section{RESULTADOSEDISCUSSÃO}

O horário interferiu no consumo de alimento, sendo que entre 8:00 e 10:00 h, o consumo foi superior ao final da manhã (entre 10:00 e 12:00 h). Assim o fornecimento do alimento nas primeiras horas do dia promove um maior consumo em menor tempo, independente do alimentador utilizado (tabela I). Os valores médios de temperatura e umidade durante o período foram de $29,32 \pm 3,30$ e $68,61 \pm 13,87^{\circ} \mathrm{C}$, respectivamente.

Alves et al. (2011) e Silva et al. (2011) observaram que abelhas mandaçaia (M. q. anthidioides) e uruçu (M. scutellaris), respectivamente, preferem forragear no período mais frio do dia, sob alta umidade relativa, possibilitando sua sobrevivência em um ambiente de clima semi-árido. Estes resultados também foram constatados nesse trabalho onde nos horários mais amenos e úmidos as abelhas realizaram o maior consumo do alimento fornecido devido ao estimulo de forrageamento.
Tabela I. Consumo de alimento por madaçaia para os três tipos de alimentadores. (Food consumption by madaçaia bee in three devices).

\begin{tabular}{lcc}
\hline Horario & Consumo $(\mathrm{mL})$ & Consumo $(\%)$ \\
\hline Eiratama $(\mathrm{n}=840)$ & & \\
8:00-9:00 & $0,65 \pm 1,40^{\mathrm{a}}$ & $2,21 \pm 4,73^{\mathrm{a}}$ \\
9:00-10:00 & $0,48 \pm 1,39^{\mathrm{ab}}$ & $1,55 \pm 4,64^{\mathrm{ab}}$ \\
10:00-11:00 & $0,27 \pm 1,08^{\mathrm{b}}$ & $0,85 \pm 3,35^{\mathrm{b}}$ \\
11:00-12:00 & $0,23 \pm 0,78^{\mathrm{b}}$ & $0,74 \pm 2,60^{\mathrm{b}}$ \\
Consumo médio & $0,41 \pm 1,20^{\mathrm{C}}$ & $1,37 \pm 3,97^{\mathrm{C}}$ \\
& & \\
Pernambucano & $(\mathrm{n}=840)$ & \\
8:00-9:00 & $3,11 \pm 4,14^{\mathrm{a}}$ & $10,24 \pm 13,74^{\mathrm{a}}$ \\
9:00-10:00 & $3,11 \pm 3,52^{\mathrm{ab}}$ & $10,23 \pm 11,67^{\mathrm{ab}}$ \\
10:00-11:00 & $2,87 \pm 3,70^{\mathrm{b}}$ & $9,38 \pm 12,32^{\mathrm{b}}$ \\
11:00-12:00 & $2,86 \pm 3,70^{\mathrm{b}}$ & $9,52 \pm 18,33^{\mathrm{b}}$ \\
Consumo médio & $2,98 \pm 4,28^{\mathrm{B}}$ & $9,88 \pm 14,23^{\mathrm{B}}$ \\
& & \\
Prato $(\mathrm{n}=840)$ & & \\
8:00-9:00 & $14,68 \pm 11,57^{\mathrm{a}}$ & $49,18 \pm 38,40^{\mathrm{a}}$ \\
9:00-10:00 & $11,14 \pm 12,01^{\text {ab }}$ & $37,28 \pm 39,97^{\text {ab }}$ \\
10:00-11:00 & $13,93 \pm 13,44^{\mathrm{b}}$ & $46,41 \pm 44,82^{\mathrm{b}}$ \\
11:00-12:00 & $14,46 \pm 14,01^{\mathrm{b}}$ & $48,15 \pm 46,75^{\mathrm{b}}$ \\
Consumo médio & $13,55 \pm 12,86^{\mathrm{A}}$ & $45,18 \pm 42,80^{\mathrm{A}}$ \\
Média geral & $5,65 \pm 9,69$ & $18,81 \pm 32,31$
\end{tabular}

abMédias com letras diferentes diferem entre si $(p=0,05)$ para o alimentador nos diferentes horários. ${ }^{A B}$ Médias com letras diferentes diferem entre si $(p=0,05)$ para os diferentes dispositivos.

O alimentador tipo Prato apresentou maior consumo, com média de 13,55 mL, seguido pelo Pernambucano e Eiratama, respectivamente (tabela I). Este resultado pode ser alcançado devido o dispositivo Prato estar localizado no interior da caixa, não teve interferência da luz, vento e ambientais, como a temperatura e nebulosidade.

Dias et al. (2008) avaliaram o uso da alimentação artificial energética à base de mel com abelhas jandaíras (M. subnitida) fornecida diretamente nos potes vazios, tendo um consumo diário médio de $8,65 \mathrm{~mL}$. Esse menor consumo diário tem relação à forma de alimentação fornecida, mostrando assim a importância no estudo de diferentes 
alimentadores e a eficiência no aproveitamento do alimento pelas abelhas.

Com relação à facilidade de manejo, o tipo Prato foi o melhor, pois o alimentador encontrava-se na parte interna (melgueira), com menor deposição de geoprópolis. Já o dispositivo Pernambucano foi o que requisitou maior tempo de coleta do alimento pelas abelhas, pois as abelhas depositavam geoprópolis fechamento da tampa do alimentador. Neste caso era necessário fazer a retirada de toda a geoprópolis, levando o tempo de 10 minutos por caixa para posterior fornecimento do alimento.

Dentre as dificuldades encontradas foi observado à presença de formigas e abelhas Apis mellifera tentando saquear o alimento no alimentador dispositivo Eiratama. Por esse motivo a tampa deste dispositivo deve ter um bom encaixe, de forma a não favorecer a entrada de inimigos. Já o Pernambucano

\section{BIBLIOGRAFIA}

Alves, R.M.O.; Souza, B.A. and Carvalho, C.A.L. 2007. Notas sobre a bionomia de Melipona mandacaia (Apidae: Meliponini). Magistra, 19: 204-212.

Alves, T.T.L.; Barbosa, R.S.; Santos, W.D.; Silva, J.N. e Neto, J.P.H. 2011. Estudo do desenvolvimento e força de trabalho de abelha mandaçaia (Melipona mandacaia) em meliponário no estado do Ceará, como ferramenta para o manejo racional da espécie. Rev Verde, 6: 163-168.

Araújo, C.S.F. e Sousa, A.N. 2011. Estudo do processo de desertificação na caatinga: uma proposta de educação ambiental. Cien Ed, 17: 975-986.

Contrera, F.A.L.; Menezes, C. and Venturieri, G.C. 2011. New horizons on stingless beekeeping (Apidae, Meliponini). Rev Bras Zootecn, 40: 4851.

Dias, A.M.; Filgueira, M.A.; Oliveira, F.L.; Costa, E.M. e Dias, V.H.P. 2010. Influência da teve ocorrência de mortalidade de abelhas, pela dificuldade das abelhas em coletar o alimento. Por outro lado, os alimentadores Eiratama e Prato não apresentaram mortalidades, não havendo preocupação para o meliponicultor com este fator.

\section{CONCLUSÕES}

O alimentador tipo Prato apresentou o maior consumo de alimento e facilidade de manejo para colônias de Melipona quadrifasciata anthidioides, alojadas em caixa modelo INPA.

\section{AGRADECIMENTOS}

Agradecemos a FAPESB, pelo recurso financeiro referente aos Termos de Outorgas PPP0064/2010 e BOL1836/2010, e ao CNPq pela bolsa referente ao processo 303237/ 2010-4.

alimentação artificial protéica no desenvolvimento de abelhas jandaira (Melipona subnitida ducke) (Apidae: Meliponini). Rev Verde, 5: 196206.

Dias, V.H.P.; Filgueira, M.A.; Oliveira, F.L.; Dias, A.M. e Costa, E.M.C. 2008. Alimentação artificial à base de mel e suas implicações no desenvolvimento de famílias de abelhas jandaíras (Melipona subnitida Ducke) em Mossoró-RN. Rev Verde, 3: 40-44.

Lima, B.G.; Coelho, M.F.B. e Oliveira, O.F. 2012. Caracterização florística de duas áreas de caatinga na região centro-sul do Ceará, Brasil. Bioscience, 28: 277-296.

Silva, M.D.; Ramalho, M. e Rosa, J.F. 2011. Porque Melipona scutellaris (Hymenoptera, Apidae) forrageia sob alta umidade relativa do ar? Iheringia, 101: 131-137.

SAS. 2007. Statistical Analysis System. SAS User's Guide, 8. ed. SAS Institute. Cary. 